\title{
Impact of the International Association for the Study of Lung Cancer/American Thoracic Society/European Respiratory Society classification of stage IA adenocarcinoma of the lung: Correlation between computed tomography images and EGFR and KRAS gene mutations
}

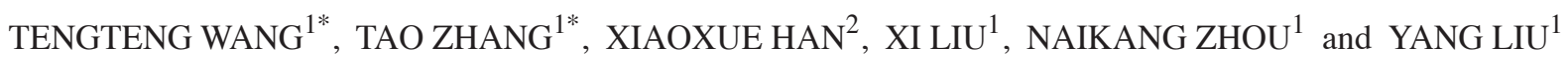 \\ ${ }^{1}$ Department of Thoracic Surgery, Chinese PLA General Hospital, Beijing 100853; ${ }^{2}$ Department of Ultrasound, \\ Chinese Capital Institute of Pediatrics, Beijing 100020, P.R. China
}

Received September 3, 2014; Accepted March 18, 2015

DOI: $10.3892 /$ etm.2015.2422

\begin{abstract}
The aim of the present study was to compare pathological diagnoses, as determined by the new International Association for the Study of Lung Cancer/American Thoracic Society/European Respiratory Society (IASLC/ATS/ERS) classification, with conventional radiological features. In addition, the present study aimed to evaluate the correlation among clinical characteristics, computed tomography (CT) images and gene mutation status in patients with stage IA adenocarcinoma of the lung. A total of 212 patients with stage IA lung adenocarcinoma were included in the study. The patients were classified into pure ground-glass opacity (pGGO), mixed GGO (mGGO) and solid GGO (sGGO) by CT imaging. Histological subtype was classified according to the IASLC/ATS/ERS classification of lung adenocarcinoma. In addition, epidermal growth factor receptor (EGFR) and Kirsten rat sarcoma (KRAS) mutation assays were performed, and $36.8 \%$ of patients $(78 / 212)$ were determined to have an EGFR mutation, while $8.5 \%$ of patients (18/212) were found to have a KRAS mutation. According to the IASLC/ATS/ERS classification, 44 cases were diagnosed as adenocarcinoma in situ (AIS; 20.8\%), 62 cases were diagnosed as minimally invasive adenocarcinoma (MIA; 29.2\%) and 106 cases were classified as invasive adenocarcinoma (IAC; $50.0 \%$ ). pGGO image patterns were observed in $39.2 \%$ of patients $(n=83)$, while $\mathrm{mGGO}$ and $\mathrm{sGGO}$ patterns were observed in $28.8 \%$
\end{abstract}

Correspondence to: Professor Naikang Zhou, Department of Thoracic Surgery, Chinese PLA General Hospital, 28 Fuxing Road, Beijing 100853, P.R. China

E-mail: wtwangteng@126.com

* Contributed equally

Key words: lung adenocarcinoma, ground-glass opacity, gene mutation $(n=61)$ and $32.0 \%(n=68)$ of patients, respectively. From pGGO to sGGO, cases of AIS and MIA were shown to have a decreasing trend, while IAC cases exhibited an increasing trend $(\mathrm{P}=0.036)$. Analysis of the correlation between $\mathrm{CT}$ image patterns and gene mutations demonstrated that L858R point mutations, exon 19 deletions and KRAS mutations were more common in lesions with a lower GGO proportion $(\mathrm{P}=0.029$, 0.027 and 0.018 , respectively). Therefore, according to the IASLC/ATS/ERS classification, GGO imaging patterns were shown to correlate with subtypes of adenocarcinomas. In addition, EGFR and KRAS mutations were found to be associated with lesions with a low GGO proportion. Therefore, analysis of GGO lesions may offer useful indications of the histological subtype of an adenocarcinoma in patients with stage IA lung adenocarcinoma, and predictive value for EGFR and KRAS mutations.

\section{Introduction}

Typical characteristics of non-small cell lung cancer (NSCLC) include the accumulation of multiple genetic alterations, resulting from the inactivation of tumor-suppressor genes, the activation of oncogenes and epigenetic changes. The transmembrane epidermal growth factor receptor (EGFR) is detected in $80-85 \%$ of patients with NSCLC, with the levels of expression varying widely on a continuous scale. The expression and activity levels of EGFR have been demonstrated to be closely associated with tumor cell survival and proliferation $(1,2)$.

EGFR mutations are associated with specific characteristics, such as adenocarcinoma histological findings, a non-smoking status, the female gender and East Asian ethnicity $(3,4)$. A previous study demonstrated that activating mutations of the EGFR gene were associated with sensitivity to EGFR tyrosine kinase inhibitors (TKIs), including gefitinib and erlotinib (5). Gefitinib and erlotinib are synthetic small molecules that are used for the treatment of patients with unresectable or recurrent NSCLC $(3,5)$. The presence of EGFR mutations is a useful predictor for the efficacy of such TKIs. Numerous 
EGFR mutations types have been reported. For example, a deletion in exon 19 and point mutations of L858R in exon 21 are representative mutations, since they comprise $\sim 90 \%$ of the mutations $(3,6)$. The prevalence of Kirsten rat sarcoma (KRAS) mutation is known to be associated with cigarette smoking (7). The mutated form of KRAS is constitutively active, which results in the transformation of immortalized cells and the promotion of cell proliferation. The KRAS mutation status is a prognostic factor for survival; patients with a KRAS mutation have been found to have a shorter survival time compared with patients with the wild-type KRAS gene (8). In addition, the KRAS mutation status is a predictor for poor therapeutic efficacy with EGFR-TKIs; however, KRAS mutations have not been shown to affect chemotherapeutic efficacy (9). EGFR and KRAS mutations appear to be mutually exclusive (10). Currently, targeted therapy is not available for patients with KRAS mutations, although mitogen-activated protein kinase kinase inhibitors are being investigated in clinical trials $(11,12)$.

In recent years, the incidence of lung adenocarcinoma, the most common histological subtype of lung cancer in the majority of the world, has been increasing (13). Despite the improvement in numerous treatment methods, the mortality rate from lung cancer has remained high for several decades (14). However, due to advances in computed tomography (CT) imaging and the widening availability of lung cancer screening with the use of spiral low-dose CT, the detection frequency of small and early lung cancers, which are not visible on chest $\mathrm{X}$-rays, is increasing. Small nodules with ground-glass opacity (GGO) in the peripheral lung can be more effectively detected, and small and early lung cancer lesions may be observed in $\mathrm{CT}$ imaging as pure GGO (pGGO), mixed GGO (mGGO) or solid patterned GGO (sGGO). Neoplastic small nodules presenting GGO include the full spectrum of preinvasive to invasive lesions, under the putative hypothesis that lung cancer develops sequentially from atypical adenomatous hyperplasia to bronchioloalveolar carcinoma (BAC) to adenocarcinoma with BAC features. However, the mechanism underlying lesion progression over time, in terms of radiological and molecular characteristics, remains unclear. In the present study, associations between CT features and the EGFR and KRAS mutation status were investigated in patients with early lung adenocarcinomas. The aim of the study was to determine whether evaluation of the CT findings may aid the determination of the presence of EGFR and KRAS mutations, particularly when a tumor specimen is unable to be obtained.

\section{Patients and methods}

Patients. This study was a retrospective cohort research and was approved by the Institutional Review Board of the Chinese PLA General Hospital (Beijing, China). All the patients provided written informed consent. Between January 2011 and January 2014, 871 patients underwent surgical resection for lung adenocarcinoma at the Chinese PLA General Hospital. Of these, 706 resected samples were available for analysis of the EGFR and KRAS mutation status. The records of the patients were reviewed to obtain further CT findings, and clinical and pathological information. The tumor, node and metastasis staging system was applied according to the 7 th edition of the tumor-node-metastasis classification of malignant tumors for lung cancer outlined by the International Association for the Study of Lung Cancer (IASLC) (15). In addition, the pathological diagnoses were based on the 2004 World Health Organization histological classification system (16) and the 2011 IASLC/American Thoracic Society (ATS)/European Respiratory Society (ERS) lung adenocarcinoma classification system (17). Patients who met the following criteria were selected for the study: i) Solitary pulmonary nodule; ii) adenocarcinoma; iii) lung tumor lesions completely resected with radical lymph node dissection; and iv) EGFR and KRAS mutation assays of tumor specimens had been performed. The excluding criteria were as follows: i) Non-adenocarcinoma cell type; ii) pathological stages IB, II, III and IV; iii) a tumor size of $>3 \mathrm{~cm}$; and iv) multiple lung cancers. A total of 212 patients were included in the study.

CT findings. Patients underwent preoperative helical CT scanning (LightSpeed ${ }^{\mathrm{TM}}$ Ultra; GE Medical Systems, Milwaukee, WI, USA) in the month prior to surgery. The scanning parameters used for the chest CT examination were as follows: Detector collimation, $1-5 \mathrm{~mm}$; beam pitch, 0.75-1.75; reconstruction thickness, $1-5 \mathrm{~mm}$; reconstruction interval, 1-5 mm; tube voltage, $120 \mathrm{kVp}$; tube current, $40 \mathrm{~mA}$; and reconstruction kernel, a high frequency algorithm. Two radiologists who specialized in lung cancer independently reviewed the CT images. Any differences in the interpretation of the nodule categorization between the two readers were resolved by discussion until a consensus was reached. The two radiologists had not been informed of the pathological reports or the EGFR gene mutation status. The image patterns of the tumors were categorized into $\mathrm{pGGO}, \mathrm{mGGO}$ or sGGO from the high-resolution CT scan. pGGO was defined as a hazy increase in lung attenuation without obscuring the underlying bronchial or vascular structures; $\mathrm{mGGO}$ was defined as GGO with a solid section occupying $<50 \%$ of the nodule; and sGGO was defined as GGO with a section occupying $>50 \%$ of the nodules on high-resolution CT.

Histopathological analysis. In total, 212 lung tissue samples were fixed in formalin, embedded in paraffin and stained with hematoxylin and eosin for histological examination using an Olympus CX31 microscope (Olympus Corporation, Tokyo, Japan). One board-certified pathologist, with 20 years of experience performing pathological diagnosis of lung cancer, who was blinded to the information regarding the EGFR mutation status, reviewed the pathological specimens and recorded the pathological subtype of each tumor according to the IASLC/ATS/ERS classification of lung adenocarcinoma (17). The categories included were as follows: i) Adenocarcinoma in situ (AIS; formerly known as BAC), which comprised a $3 \mathrm{~cm}$ nodule, lepidic growth and mucinous, non-mucinous or mixed mucinous/non-mucinous types; ii) minimally invasive adenocarcinoma (MIA), in which the nodules were $\leq 3 \mathrm{~cm}$ with $\leq 5 \mathrm{~mm}$ of invasion, lepidic growth and mucinous, non-mucinous or mixed mucinous/non-mucinous types; iii) invasive adenocarcinoma with a predominant growth pattern of lepidic growth, in which $>5 \mathrm{~mm}$ of invasion was observed, with acinar, papillary, micropapillary or solid types with mucin; and iv) invasive adenocarcinoma variants, such as mucinous adenocarcinoma, colloid, fetal and enteric 
morphologies. The latter two categories were classified as invasive adenocarcinoma (IAC) as its own class.

Gene mutation analysis. Formalin-fixed paraffin-embedded (FFPE) tissues were analyzed to determine the EGFR and KRAS mutation status. Samples considered suitable for downstream biomarker analysis were progressed to biomarker analysis on the basis of quality, sample source and tumor content ( $>100$ tumor cells). The samples underwent a central, histopathological review to ensure that they were adequate for use and where appropriate, hematoxylin and eosin-stained tissue was classified by suitably qualified pathologists. DNA was extracted from the samples using a QIAamp DNA FFPE tissue kit (Qiagen, Hilden, Germany), according to the manufacturer's instructions. DNA was quantified to a final concentration of $2 \mathrm{ng} / \mu \mathrm{l}$ using a NanoDrop2000 spectrophotometer (NanoDrop; Thermo Fisher Scientific, Wilmington, DE, USA). EGFR mutations at exons 18 to 21 and KRAS mutations at codons 12,13 and 61 were analyzed with an amplification-refractory mutation system using AmoyDx EGFR 29 and KRAS fluorescence polymerase chain reaction (PCR) diagnostic kits (AmoyDx, Xiamen, China), according to the manufacturer's instructions. Quantitative PCR amplification was performed using an Mx3000P ${ }^{\text {TM }}$ quantitative PCR system (Stratagene; Agilent Technologies, Inc., Santa Clara, CA, USA). PCR cycling conditions were as follows: Initial denaturation at $95^{\circ} \mathrm{C}$ for $5 \mathrm{~min}$, followed by 15 cycles of denaturation at $95^{\circ} \mathrm{C}$ for $25 \mathrm{sec}$, annealing at $64^{\circ} \mathrm{C}$ for $20 \mathrm{sec}$ and elongation at $70^{\circ} \mathrm{C}$ for $20 \mathrm{sec}$; and 31 cycles at $95^{\circ} \mathrm{C}$ for $25 \mathrm{sec}$, $60^{\circ} \mathrm{C}$ for $35 \mathrm{sec}$ and $72^{\circ} \mathrm{C}$ for $20 \mathrm{sec}$. FAM and HEX fluorescence signals were collected in the third step. The primers used are under patent; no. ZL2009101114992 for the EGFR kit and no. ZL2009101115016 for the KRAS kit. Data were analyzed using MxPro software (version 4.10; Stratagene; Agilent Technologies, Inc.). Relative expression levels were determined using the cycle threshold $(\mathrm{Ct})$, which was the cycle number when the strength value of the fluorescence signal was greater than the background signal. $\Delta \mathrm{Ct}$ values were equal to the $\mathrm{Ct}$ value of the sample minus the control.

Statistical analysis. The $\chi^{2}$ test or Fisher's exact test were used for comparisons of categorical variables. $\mathrm{P}<0.05$ was considered to indicate a statistically significant difference, and all reported P-values were two-sided. Statistical analyses were performed using SPSS for Windows (version 19.0; IBM SPSS, Armonk, NY, USA).

\section{Results}

Clinical information and types of gene mutation. Clinical features, pathological characteristics, radiological findings and EGFR and KRAS gene mutation analyses are summarized in Table I. The ages of the 212 patients ranged between 36 and 76 years, and the median age was 58 years. With regard to the smoking habits, 97 patients $(45.8 \%)$ had never smoked, 85 individuals $(40.1 \%)$ were current smokers and 30 patients $(14.2 \%)$ were former smokers. Former smokers were defined as patients who had quit smoking $\geq 1$ year prior to surgery (18). According to the IASLC/ATS/ERS classification, 44 patients were diagnosed with AIS (20.8\%), 62 cases were classified
Table I. Summary of clinical factors and gene mutation status.

\begin{tabular}{|c|c|}
\hline Variables & Cases, n (\%) \\
\hline Median age, years (range) & $58(36-76)$ \\
\hline \multicolumn{2}{|l|}{ Gender } \\
\hline Male & $130(61.3)$ \\
\hline Female & $82(38.7)$ \\
\hline \multicolumn{2}{|l|}{ Smoking history } \\
\hline Never & $97(45.8)$ \\
\hline Former $\mathrm{a}$ current & $115(54.3)$ \\
\hline \multicolumn{2}{|l|}{ Pathological diagnosis } \\
\hline AIS & $44(20.8)$ \\
\hline MIA & $62(29.2)$ \\
\hline IAC & $106(50.0)$ \\
\hline \multicolumn{2}{|l|}{ Image patterns } \\
\hline pGGO & $83(39.2)$ \\
\hline mGGO & $61(28.8)$ \\
\hline sGGO & $68(32.0)$ \\
\hline \multicolumn{2}{|l|}{ EGFR mutation status } \\
\hline Wild type & $134(63.2)$ \\
\hline L858R & $34(16.0)$ \\
\hline Exon 19 deletion & $39(18.4)$ \\
\hline Others & $5(2.4)$ \\
\hline \multicolumn{2}{|l|}{ KRAS mutation status } \\
\hline Wild type & $194(91.5)$ \\
\hline Mutation & $18(8.5)$ \\
\hline
\end{tabular}

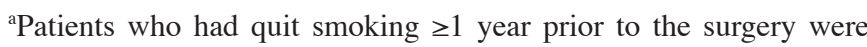
defined as former smokers. AIS, adenocarcinoma in situ; MIA, minimally invasive adenocarcinoma; IAC, invasive adenocarcinoma; GGO, ground-glass opacity; p, pure; m, mixed; s, solid; EGFR, epidermal growth factor receptor; KRAS, Kirsten rat sarcoma.

as MIA (29.2\%) and 106 patients were diagnosed with IAC (50.0\%). A pGGO pattern from the CT scan was recorded in $39.2 \%$ of cases $(n=83)$, while an mGGO pattern was observed in $28.8 \%$ of cases $(n=61)$ and an sGGO pattern was observed in $32.0 \%$ of cases $(n=68)$. The EGFR mutation rate was $36.8 \%$ $(\mathrm{n}=78)$, in which 39 cases had an exon 19 deletion $(18.4 \%)$, 34 cases exhibited a L858R point mutation (16.0\%) and five cases $(2.4 \%)$ were identified as other mutation types. The KRAS mutation rate was $8.5 \%(n=18)$. Table II shows the types of EGFR and KRAS mutations. The majority of EGFR mutations were due to a point mutation of L858R in exon $21(n=34$, 43.6\%) and a E746_A750 deletion in exon $19(n=22,28.2 \%)$. All types of KRAS mutation occurred at codons 12 and 13. Two G12R mutations (11.1\%), three G12C mutations (16.7\%), five G12V mutations (27.8\%), six G12D mutations (33.3\%) and two G13D mutations (11.1\%) were identified.

Image patterns and pathological diagnosis. Associations between image patterns and pathological diagnosis are shown in Table III. AIS was found in $25.3 \%$ (21/83) of the pGGO group, $21.3 \%$ (13/61) of the mGGO group and $14.7 \%(10 / 68)$ of the sGGO group. MIA was observed in $36.1 \%(30 / 83)$ of the 
Table II. Information on the gene mutation status.

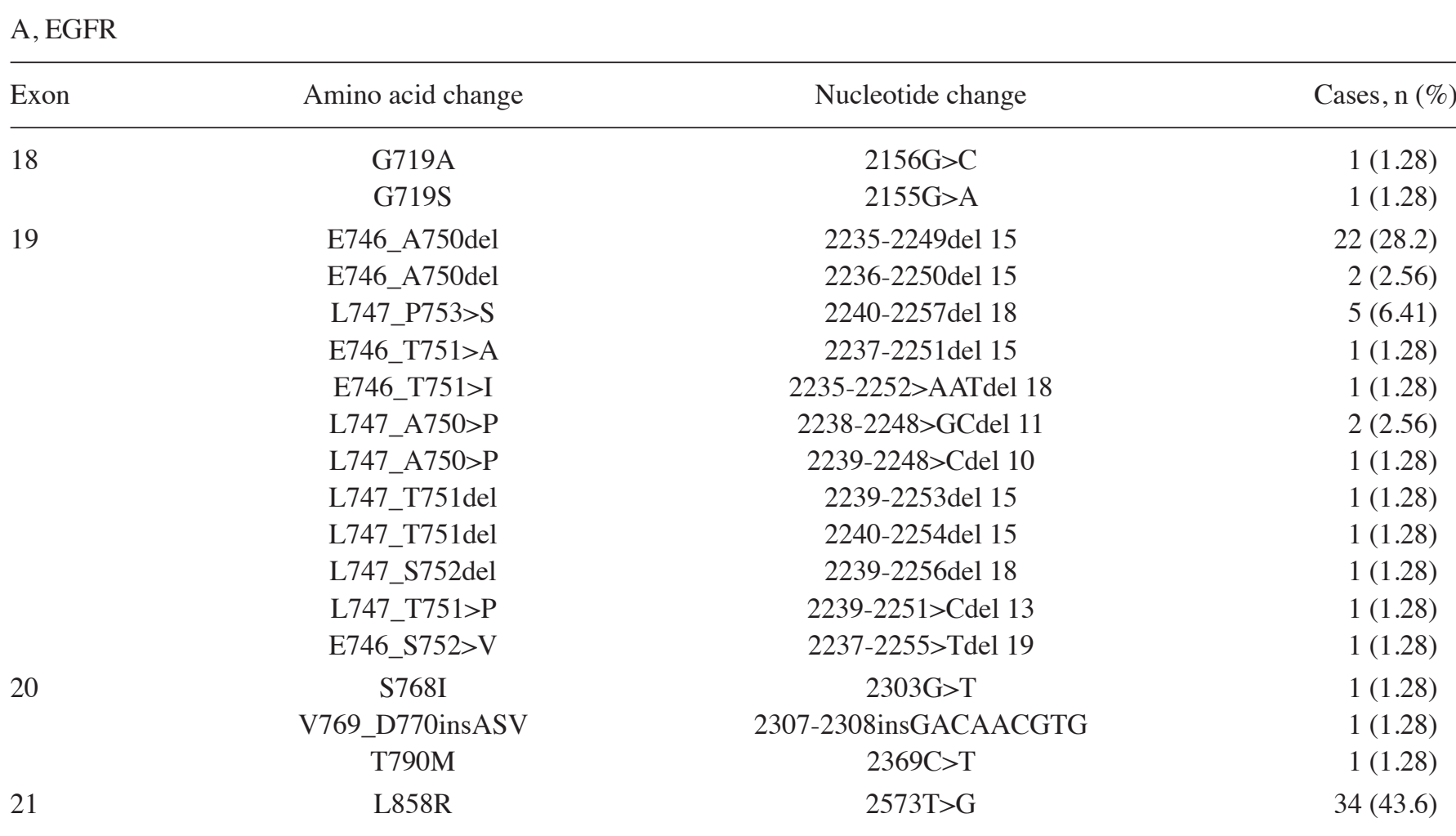

\section{B, KRAS}

\begin{tabular}{lccr}
\hline Exon & Amino acid change & Nucleotide change & Cases, $\mathrm{n}(\%)$ \\
\hline 2 & G12R & $34 \mathrm{G}>\mathrm{C}$ & $2(11.1)$ \\
G12C & $34 \mathrm{G}>\mathrm{T}$ & $5(27.8)$ \\
G12V & $35 \mathrm{G}>\mathrm{T}$ & $6(33.3)$ \\
G12D & $35 \mathrm{G}>\mathrm{A}$ & $2(11.1)$ \\
\hline
\end{tabular}

EGFR, epidermal growth factor receptor; KRAS, Kirsten rat sarcoma virus.

Table III. Image patterns and pathological diagnosis.

\begin{tabular}{lcccc}
\hline & \multicolumn{3}{c}{ Pathological diagnosis } & \\
\cline { 2 - 4 } $\begin{array}{l}\text { Image } \\
\text { patterns }\end{array}$ & AIS, n(\%) & MIA, n (\%) & IAC, n(\%) & Total \\
\hline pGGO & $21(25.3)$ & $30(36.1)$ & $32(38.6)$ & 83 \\
mGGO & $13(21.3)$ & $18(29.5)$ & $30(49.2)$ & 61 \\
sGGO & $10(14.7)$ & $14(20.6)$ & $44(64.7)$ & 68 \\
Total & 44 & 62 & 106 & 212
\end{tabular}

AIS, adenocarcinoma in situ; MIA, minimally invasive adenocarcinoma; IAC, invasive adenocarcinoma; GGO, ground-glass opacity; p, pure; m, mixed; s, solid.

pGGO group, $29.5 \%$ (18/61) of the mGGO group and $20.6 \%$ $(14 / 68)$ of the sGGO group. Furthermore, IAC was found in
$38.6 \%(32 / 83)$ of the pGGO group, $49.2 \%(30 / 61)$ of the mGGO group and $64.7 \%$ (44/68) of the sGGO group. Therefore, AIS and MIA were shown to exhibit a decreasing trend with regard to the solid section of the tumors, whereas IAC exhibited an increasing trend. The $\chi^{2}$ test was performed $\left(\chi^{2}=10.254\right)$ and the differences were determined to be statistically significant $(\mathrm{P}=0.036)$. Thus, the solid portion of the $\mathrm{CT}$ image patterns and the histopathological invasion type may be well-matched (Figs. 1-3).

Clinical factors and gene mutation status. Table IV shows the patient characteristics and gene mutation status. L858R point mutations and exon 19 deletions were more frequent in females than in males, as compared with the wild-type EGFR $(\mathrm{P}<0.05)$. However, no statistically significant difference was observed between genders with regard to the KRAS mutation and wild type genotypes $(\mathrm{P}=0.985)$. In patients with no history of smoking, L858R point mutations were more frequently 
A

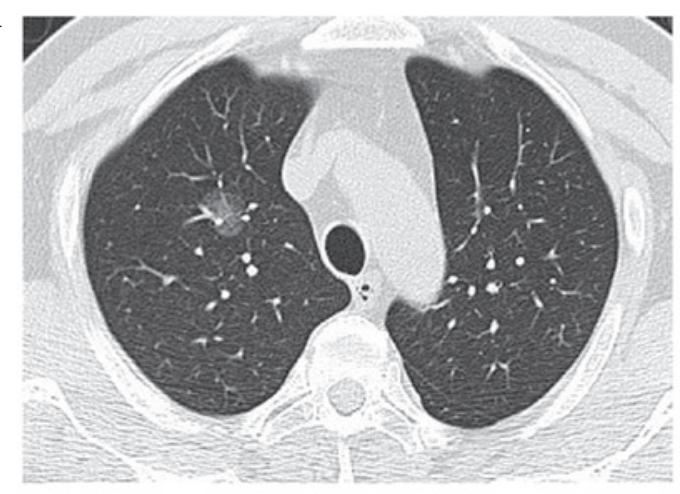

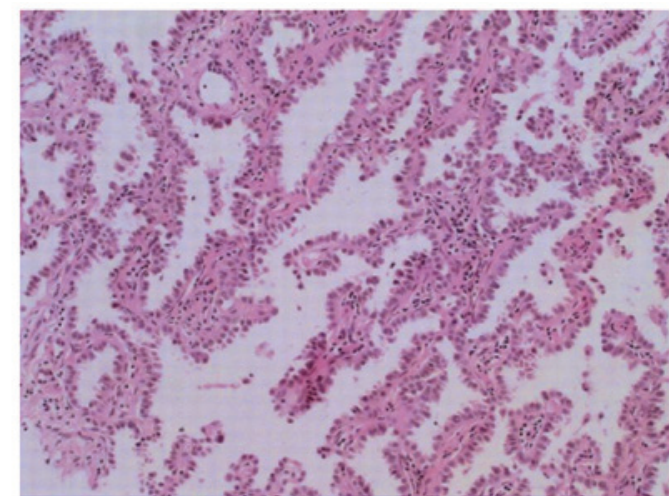

Figure 1. (A) Computed tomography image showing a tumor with pure ground-glass opacity, without obscuring the underlying vascular structures. (B) Lesion was diagnosed as a adenocarcinoma in situ (hematoxylin and eosin stain; magnification, x200). The findings were negative for epidermal growth factor receptor and Kirsten rat sarcoma mutations.

A

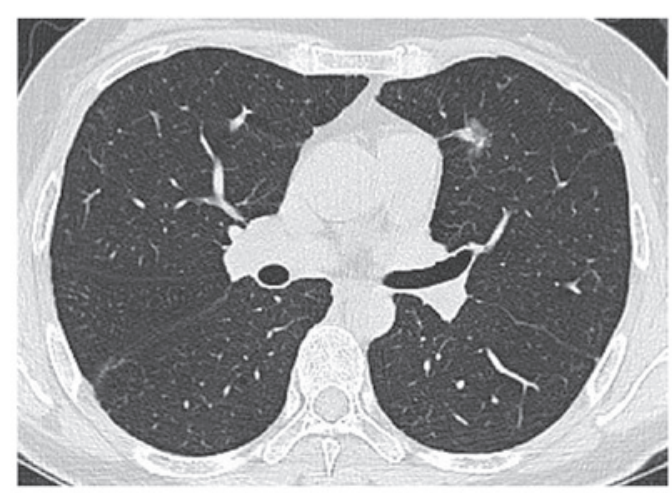

B

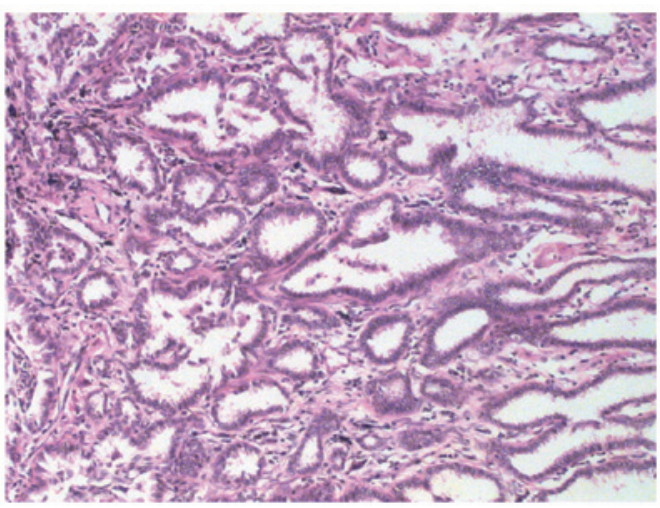

Figure 2. (A) Computed tomography image showing a tumor with mixed ground-glass opacity (GGO), with a tiny solid component and peripheral GGO. (B) Lesion was diagnosed as a minimally invasive adenocarcinoma (hematoxylin and eosin stain; magnification, $\mathrm{x} 200$ ). The findings were negative for epidermal growth factor receptor and Kirsten rat sarcoma mutations.

A

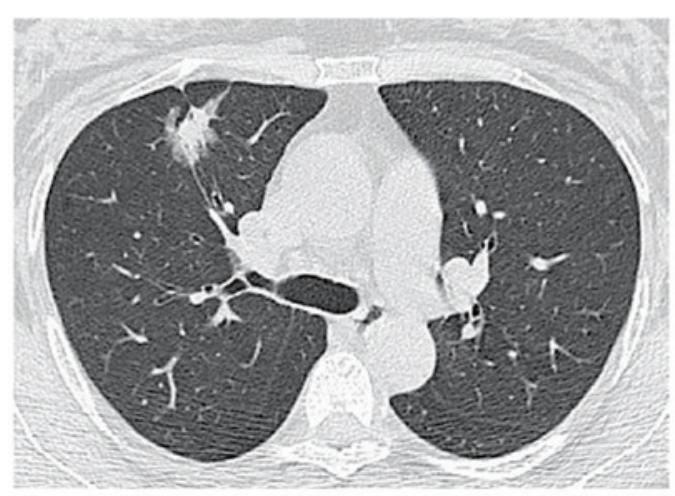

B

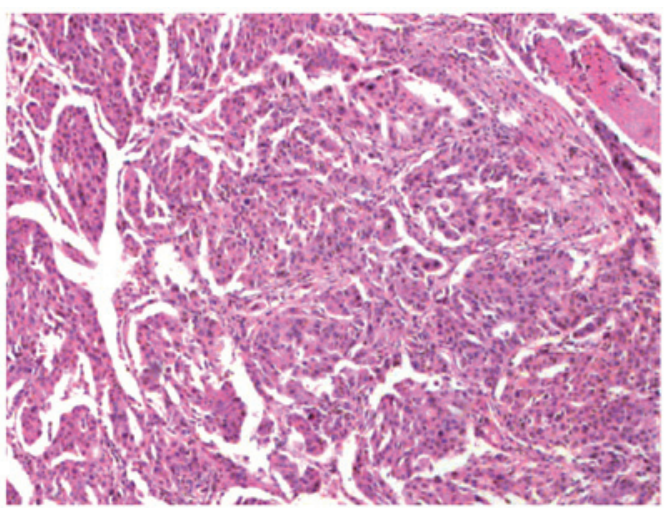

Figure 3. (A) Computed tomography image showing a tumor with solid ground-glass opacity (GGO), with a central solid component and peripheral GGO. (B) Lesion was diagnosed as an invasive adenocarcinoma (hematoxylin and eosin stain; magnification, $\mathrm{x} 200$ ). The findings indicated an epidermal growth factor receptor mutation, but no Kirsten rat sarcoma mutation.

observed compared with former/current smokers; however, smoking history was not shown to correlate with an exon 19 deletion ( $\mathrm{P}=0.067)$. In addition, in patients with a history of smoking, KRAS mutations were observed more frequently compared with non-smokers $(\mathrm{P}=0.010)$. The rate of an L858R point mutation was $7 / 44(15.9 \%)$ in patients with AIS, 8/62 $(12.9 \%)$ in MIA patients and 19/106 (17.9\%) in patients with IAC. Furthermore, the rate of an exon 19 deletion was $4 / 44$
(9.1\%) in AIS patients, 10/62 (16.1\%) in patients with MIA and 25/106 (23.6\%) in IAC patients. The rate of a KRAS mutation was 2/44 (4.5\%) in patients with AIS, 5/62 (8.1\%) in MIA patients and 11/106 (10.4\%) in patients with IAC. Histological diagnosis was shown to have an association with an exon 19 deletion $(\mathrm{P}=0.041)$; however, the trend association was not observed for the L858R point mutation or KRAS mutation status $(\mathrm{P}=0.411$ and 0.501 , respectively), although there was 
Table IV. Clinical factors and gene mutation status.

\begin{tabular}{|c|c|c|c|c|c|c|c|c|}
\hline \multirow[b]{2}{*}{ Clinical factors } & \multicolumn{3}{|c|}{ EGFR, n } & \multicolumn{2}{|c|}{ KRAS, n } & \multirow[b]{2}{*}{ P1 } & \multirow[b]{2}{*}{$\mathrm{P} 2$} & \multirow[b]{2}{*}{ P3 } \\
\hline & $\begin{array}{l}\text { Wild type } \\
(\mathrm{n}=134)\end{array}$ & $\begin{array}{l}\text { L858R } \\
(n=34)\end{array}$ & $\begin{array}{c}\text { Exon } 19 \\
\text { deletion }(n=39)\end{array}$ & $\begin{array}{l}\text { Wild type } \\
(\mathrm{n}=194)\end{array}$ & $\begin{array}{l}\text { Mutation } \\
(\mathrm{n}=18)\end{array}$ & & & \\
\hline \multicolumn{9}{|l|}{ Gender } \\
\hline Male & 100 & 13 & 15 & 119 & 11 & $<0.001$ & $<0.001$ & 0.985 \\
\hline Female & 34 & 21 & 24 & 75 & 7 & & & \\
\hline \multicolumn{9}{|l|}{ Smoking history } \\
\hline Never & 47 & 27 & 20 & 94 & 3 & $<0.001$ & 0.067 & 0.010 \\
\hline Former ${ }^{\mathrm{a}}$ current & 87 & 7 & 19 & 100 & 15 & & & \\
\hline \multicolumn{9}{|c|}{ Histological diagnosis } \\
\hline AIS & 33 & 7 & 4 & 42 & 2 & 0.411 & 0.041 & 0.501 \\
\hline MIA & 44 & 8 & 10 & 57 & 5 & & & \\
\hline IAC & 57 & 19 & 25 & 95 & 11 & & & \\
\hline \multicolumn{9}{|l|}{ Image pattern } \\
\hline pGGO & 62 & 8 & 11 & 80 & 3 & 0.029 & 0.027 & 0.018 \\
\hline mGGO & 39 & 11 & 10 & 57 & 4 & & & \\
\hline sGGO & 33 & 15 & 18 & 57 & 11 & & & \\
\hline
\end{tabular}

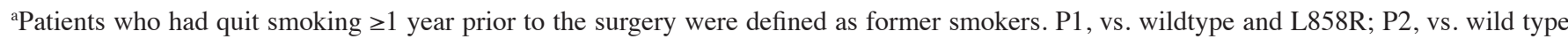
and exon 19; P3, vs. wild type and Mutation KRAS. AIS, adenocarcinoma in situ; MIA, minimally invasive adenocarcinoma; IAC, invasive adenocarcinoma; GGO, ground-glass opacity; p, pure; m, mixed; s, solid; EGFR, epidermal growth factor receptor; KRAS, Kirsten rat sarcoma.

a tendency. A statistically significant association was identified between the image pattern and gene mutation status. As the proportion of GGO decreased (from pGGO to $\mathrm{sGGO}$ ), the frequency of L858R point mutations, exon 19 deletions and KRAS mutations increased $(\mathrm{P}=0.029,0.027$ and 0.018 , respectively).

\section{Discussion}

Lung cancer remains the leading cause of cancer mortality worldwide, with late diagnosis one of the major challenges to improving outcomes $(19,20)$. Preliminary trials using spiral (helical) low-dose CT for lung cancer screening have produced promising results, with stage I lung cancer detected in $>80 \%$ of newly diagnosed cases (21-23). In recent years, the National Comprehensive Cancer Network have recommended lung cancer screening using low-dose helical CT for selected high-risk patients who are current or former smokers (24). Increasingly, small nodules have been identified. In the present study, tumors based on the proportion of the solid section in the nodules were divided into three groups, namely pGGO (non-solid part), mGGO (solid part of $<50 \%$ ) and sGGO (solid part of $\geq 50 \%$ ). The proportion of the solid section is usually associated with disease progression (25). Previous studies have reported that the solid proportion in advanced-stage lesions is significantly larger compared with those in lesions at an earlier stage $(25,26)$. In addition, a number of studies have found that a low proportion of solid matter in adenocarcinoma is a good prognostic indicator (25-28). Therefore, the solid portion of a GGO nodule in lung adenocarcinoma increases the biological invasion of the tumor, which indicates that a solid section increases the level of suspicion of invasive adenocarcinoma. In the present study, the image pattern of GGO was shown to correlate with the IASLC/ATS/ERS histological subtypes of adenocarcinoma. According to this pathological-radiological correlation, the majority of AIS and MIA cases corresponded to pGGO, while IAC cases corresponded to sGGO.

EGFR and KRAS mutations are two of the most common mutations in NSCLC $(29,30)$. The presence of EGFR mutations is a critical biological determinant for adequate therapy selection in patients with lung cancer (31). A significant association has been identified between EGFR mutations, particularly exon 19 deletions and exon 21 (L858R) and exon 18 mutations, and a sensitivity to TKIs (4,32-34). In addition, exon 20 insertion mutations have been hypothesized to be a valuable predictor of resistance to clinically achievable levels of TKIs $(35,36)$. The prevalence of EGFR mutations in Asian populations with an advanced stage of lung adenocarcinoma is up to $51.4 \%$ (37). In the present study, patients with stage IA lung adenocarcinoma, which is known to have an improved prognosis compared with the more advanced stages, were found to have a lower prevalence of EGFR mutation (36.8\%), as compared with advanced lung adenocarcinoma. The most common EGFR mutations identified in patients with NSCLC are deletions in exon 19 and mutations in exon $21(38,39)$, which concurs with the results of the current study. Furthermore, the present study found that the E746_A750 deletion was the most common exon 19 deletion. In the present study, the rate of a KRAS mutation was $8.4 \%$, which was lower than the KRAS mutation rate of $\sim 25 \%$ reported in a North American population $(8,9)$. All types of KRAS mutations occurred in codons 12 and 13, with mutation at codon 12 more frequent compared with codon 13. 
In the present study, L858R point mutations and exon 19 deletions were more prevalent in females. In addition, L858R point mutations were shown to correlate with a non-smoking history. Although the difference in smoking history between individuals with exon 19 deletion and wild type genotypes was not statistically significant $(\mathrm{P}=0.067)$, a greater number of exon 19 deletions occurred in those who had never smoked. Tam et al (40) reported that the rate of KRAS mutation was $5.3 \%$ in women, which was lower compared with the $16.7 \%$ rate in men $(\mathrm{P}=0.009)$; however, Marks et al $(41)$ reported KRAS mutation rates of $15.3 \%$ in women and $19.2 \%$ in men, and observed no differences between genders $(\mathrm{P}=0.581)$. In the present study, KRAS mutations were not found to correlate with gender, although a correlation was observed with the smoking status. Furthermore, the correlation between IASLC/ATS/ERS histological subtypes of adenocarcinoma and EGFR/KRAS mutations was analyzed. The rates of EGFR mutations in patients diagnosed with AIS, MIA and IAC were 25.0, 29.0 and 63.6\%, respectively. Thus, EGFR mutations were more frequently observed in patients with IAC compared with those diagnosed with AIS or MIA $(\mathrm{P}=0.047)$. Although EGFR mutations have been implicated in the early stages of lung adenocarcinoma development, not all cases of AIS, MIA and IAC have an EGFR mutation. Further analysis of the L858R point mutation and exon 19 deletion indicated that exon 19 deletions were significantly associated with histological diagnosis $(\mathrm{P}=0.041)$; however, no such association was observed for L858R point mutations. Exon 19 deletions were found to be significantly associated with the histological diagnosis $(\mathrm{P}=0.041)$; however, an association was not observed for L858R point mutations. Thus, the results of the present study demonstrated that the frequency of exon 19 deletions increased with different histologies from AIS to MIA to IAC. Histologically, it may be inferred that early adenocarcinoma with an exon 19 deletion may have an aggressive behavior.

A number of studies have reported an association between EGFR and KRAS mutations with image patterns. As early as 2006, Yano et al (42) reported that EGFR mutations were more frequently detected in small peripheral adenocarcinomas with a high ratio of GGO components, as compared with those with a low ratio of GGO components. Subsequently, in 2007, Yoshida et al (43) reported that EGFR mutations exhibited no correlation with the appearance or increase in consolidation within pGGO. In 2009, Chung et al (44) examined 56 pulmonary nodules presented with GGO from 24 patients to analyze the mutation status of the EGFR and KRAS genes and any pathological-radiological correlations. The authors found that the rate of EGFR mutation was $38.4 \%$ in pGGO cases, $41.6 \%$ in mGGO cases and $50 \%$ in sGGO cases, while only two KRAS mutations were identified in sGGO cases. However, in 2010, Glynn et al (45) reported that the high proportion of GGO on a CT image was not significantly associated with the presence of an EGFR mutation or with the presence of a KRAS mutation. In 2009, Park et al (46) examined the EGFR gene copy number status in 132 patients using fluorescence in situ hybridization. The authors reported that a low EGFR gene copy number was more commonly observed in adenocarcinoma with a GGO of $>50 \%$. In 2013, Lee et al (47) reported that tumors with a GGO proportion of $>50 \%$ were less frequent among tumors with EGFR-overexpression compared with tumors without
EGFR-overexpression in 214 patients with stage I NSCLC. Furthermore, in 2011, Hsu et al (48) retrospectively surveyed 162 patients with stage I lung adenocarcinoma with a tumor size of $<3 \mathrm{~cm}$, and found that the part-solid and solid pattern tumors had more typical EGFR mutations compared with the pGGO tumors. In 2012, Aoki et al (49) analyzed 25 lung adenocarcinomas of $<3 \mathrm{~cm}$ in diameter and found a high incidence of EGFR mutations in the GGO-dominant lung adenocarcinomas; however, no correlation was observed between EGFR mutations and changes in the CT patterns. Prior to 2010, the reported correlations between EGFR and KRAS mutations with GGO image patterns were tentative. With the emergence of large sample research (48), the majority of studies have reported that a low GGO component is associated with EGFR mutations or overexpression, which is consistent with the results of the present study; however, the association between KRAS mutations and radiological findings remains unclear.

In the present study, the results demonstrated that the lower the proportion of the GGO component, the higher the frequency of EGFR or KRAS mutations in patients with stage IA adenocarcinoma of the lung. This association may be significant in diagnosis, since a low proportion of GGO correlates with a poor survival rate (25-28), and EGFR amplification or KRAS mutation are associated with a worse prognosis $(2,8)$. In addition, these observations indicate that such mutations have an association with the progressive behavior of GGO. However, it is well known that EGFR and KRAS mutations are mutually exclusive in patients with lung cancer $(10,50)$. Therefore, there can be correlation without causation between the two mutations and a low GGO proportion. Consolidation of GGO lesions may be due to unmeasured or unknown factors. Two previous studies reported that the inactivation of p53 may be associated with the appearance of central consolidation within pGGO $(43,49)$.

In conclusion, in stage IA lung adenocarcinoma patients, EGFR mutations were shown to have a relatively low incidence, as compared with more advanced stages of the disease. According to the IASLC/ATS/ERS classification, image patterns of GGO were shown to correlate with subtypes of adenocarcinomas, including AIS, MIA and IAC. Furthermore, exon 19 deletions were more frequently observed in IAC cases when compared with AIS or MIA cases; however, no association was observed with L858R point mutations. In addition, KRAS mutations were not shown to have a statistically significant association with the subtypes of adenocarcinoma. EGFR and KRAS mutations were associated with a low GGO proportion in the lesions, although further research is required to determine whether this association is causal. Therefore, evaluating the GGO image patterns of stage IA lung adenocarcinoma patients may provide important information for the subtypes of lung adenocarcinoma and the status of EGFR and KRAS mutations.

\section{References}

1. Cappuzzo F, Gregorc V, Rossi E, et al: Gefitinib in pretreated non-small-cell lung cancer (NSCLC): Analysis of efficacy and correlation with HER 2 and epidermal growth factor receptor expression in locally advanced or metastatic NSCLC. J Clin Oncol 21: 2658-2663, 2003. 
2. Cappuzzo F, Hirsch FR, Rossi E, et al: Epidermal growth factor receptor gene and protein and gefitinib sensitivity in non-small-cell lung cancer. J Natl Cancer Inst 97: 643-655, 2005.

3. Lynch TJ, Bell DW, Sordella R, et al: Activating mutations in the epidermal growth factor receptor underlying responsiveness of non-small-cell lung cancer to gefitinib. N Engl J Med 350: 2129-2139, 2004

4. Paez JG, Jänne PA, Lee JC, et al: EGFR mutations in lung cancer: correlation with clinical response to gefitinib therapy. Science 304: 1497-1500, 2004.

5. Pao W and Miller VA: Epidermal growth factor receptor mutations, small-molecule kinase inhibitors, and non-small-cell lung cancer: current knowledge and future directions. J Clin Oncol 23: 2556-2568, 2005.

6. Kosaka T, Yatabe Y, Endoh H, Kuwano H, Takahashi T and Mitsudomi T: Mutations of the epidermal growth factor receptor gene in lung cancer: biological and clinical implications. Cancer Res 64: 8919-8923, 2004.

7. Slebos RJ, Hruban RH, Dalesio O, Mooi WJ, Offerhaus GJ and Rodenhuis S: Relationship between K-ras oncogene activation and smoking in adenocarcinoma of the human lung. J Natl Cancer Inst 83: 1024-1027, 1991.

8. Tsao MS, Aviel-Ronen S, Ding K, et al: Prognostic and predictive importance of p53 and RAS for adjuvant chemotherapy in non small-cell lung cancer. J Clin Oncol 25: 5240-5247, 2007.

9. Eberhard DA, Johnson BE, Amler LC, et al: Mutations in the epidermal growth factor receptor and in KRAS are predictive and prognostic indicators in patients with non-small-cell lung cancer treated with chemotherapy alone and in combination with erlotinib. J Clin Oncol 23: 5900-5909, 2005.

10. Febbo PG, Ladanyi M, Aldape KD, et al: NCCN Task Force report: Evaluating the clinical utility of tumor markers in oncology. J Natl Compr Canc Netw 9 (Suppl 5): S1-S32, 2011.

11. Sequist LV, Heist RS, Shaw AT, et al: Implementing multiplexed genotyping of non-small-cell lung cancers into routine clinical practice. Ann Oncol 22: 2616-2624, 2011.

12. Jänne PA, Shaw AT, Pereira JR, et al: Selumetinib plus docetaxel for KRAS-mutant advanced non-small-cell lung cancer: a randomised, multicentre, placebo-controlled, phase 2 study. Lancet Oncol 14: 38-47, 2013.

13. Shin HR, Masuyer E, Ferlay J and Curado MP; Asian Contributors to CI5 IX4: Cancer in Asia - Incidence rates based on data in cancer incidence in five continents IX (1998-2002). Asian Pac J Cancer Prev 11 (Suppl 2): 11-16, 2010.

14. Alberg AJ, Ford JG and Samet JM; American College of Chest Physicians: Epidemiology of lung cancer: ACCP evidence-based clinical practice guidelines (2nd edition). Chest 132 (Suppl 3): 29S-55S, 2007.

15. Ainbinder DJ, Esmaeli B, Groo SC, Finger PT and Brooks JP Introduction of the 7 th edition eyelid carcinoma classification system from the American Joint Committee on Cancer-International Union Against Cancer staging manual. Arch Pathol Lab Med 133: 1256-1261, 2009.

16. Beasley MB, Brambilla E and Travis WD: The 2004 World Health Organization classification of lung tumors. Semin Roentgenol 40: 90-97, 2005.

17. Travis WD, Brambilla E, Noguchi M, et al: International Association for the Study of Lung Cancer/American Thoracic Society/European Respiratory Society international multidisciplinary classification of lung adenocarcinoma. J Thorac Oncol 6: 244-285, 2011.

18. Takano T, Ohe Y, Sakamoto H, et al: Epidermal growth factor receptor gene mutations and increased copy numbers predict gefitinib sensitivity in patients with recurrent non-small-cell lung cancer. J Clin Oncol 23: 6829-6837, 2005.

19. Carney DN: Lung cancer - time to move on from chemotherapy N Engl J Med 346: 126-128, 2002.

20. Chute JP, Chen T, Feigal E, Simon R and Johnson BE: Twenty years of phase III trials for patients with extensive-stage small-cell lung cancer: perceptible progress. J Clin Oncol 17: 1794-1801, 1999.

21. Henschke CI, McCauley DI, Yankelevitz DF, et al: Early lung cancer action project: Overall design and findings from baseline screening. Lancet 354: 99-105, 1999.

22. Henschke CI, Naidich DP, Yankelevitz DF, et al: Early lung cancer action project: Initial findings on repeat screenings. Cancer 92: 153-159, 2001.

23. Kaneko M, Kusumoto M, Kobayashi T, et al: Computed tomography screening for lung carcinoma in Japan. Cancer 89 (Suppl): 2485-2488, 2000.
24. National Comprehensive Cancer Network, Inc. (NCCN): Lung Cancer Screening. Version 1.2012. NCCN, Fort Washington, USA, 2011. http://www.lungcanceralliance.org/assets/docs/news/ NCCN\%20Screening\%20Guidelines\%2010_11.pdf.

25. Takashima S, Maruyama $\mathrm{Y}$, Hasegawa $\mathrm{M}$, et al: $\mathrm{CT}$ findings and progression of small peripheral lung neoplasms having a replacement growth pattern. AJR Am J Roentgenol 180: 817-826, 2003.

26. Kuriyama K, Seto M, Kasugai T, et al: Ground-glass opacity on thin-section CT: value in differentiating subtypes of adenocarcinoma of the lung. AJR Am J Roentgenol 173: 465-469, 1999.

27. Aoki T, Tomoda Y, Watanabe H, et al: Peripheral lung adenocarcinoma: correlation of thin-section CT findings with histologic prognostic factors and survival. Radiology 220: 803-809, 2001

28. Kodama K, Higashiyama M, Yokouchi H, et al: Prognostic value of ground-glass opacity found in small lung adenocarcinoma on high-resolution CT scanning. Lung Cancer 33: 17-25, 2001.

29. Carneiro JG, Couto PG, Bastos-Rodrigues L, et al: Spectrum of somatic EGFR, KRAS, BRAF, PTEN mutations and TTF-1 expression in Brazilian lung cancer patients. Genet Res (Camb) 96: e002, 2014.

30. Das BR, Bhaumik S, Ahmad F, et al: Molecular spectrum of somatic EGFR and KRAS gene mutations in non small cell lung carcinoma: Determination of frequency, distribution pattern and identification of novel variations in Indian patients. Pathol Oncol Res: Jan 31, 2015 (Epub ahead of print).

31. National Comprehensive Cancer Network, Inc. (NCCN): Non-Small Cell Lung Cancer. Version 2.2013. NCCN, Fort Washington, USA, 2013. http://www.tri-kobe.org/nccn/guideline/ lung/english/non_small.pdf.

32. Cappuzzo F, Finocchiaro G, Metro G, et al: Clinical experience with gefitinib: an update. Crit Rev Oncol Hematol 58: 31-45, 2006.

33. Sequist LV, Joshi VA, Jänne PA, et al: Response to treatment and survival of patients with non-small cell lung cancer undergoing somatic EGFR mutation testing. Oncologist 12: 90-98, 2007.

34. Ji H, Li D, Chen L, et al: The impact of human EGFR kinase domain mutations on lung tumorigenesis and in vivo sensitivity to EGFR-targeted therapies. Cancer Cell 9: 485-495, 2006.

35. Lund-Iversen M, Kleinberg L, Fjellbirkeland L, Helland $\AA$ and Brustugun OT: Clinicopathological characteristics of 11 NSCLC patients with EGFR-exon 20 mutations. J Thorac Oncol 7: 1471-1473, 2012.

36. Yasuda H, Kobayashi S and Costa DB: EGFR exon 20 insertion mutations in non-small-cell lung cancer: preclinical data and clinical implications. Lancet Oncol 13: e23-e31, 2012.

37. Shi Y, Au JS, Thongprasert S, et al: A prospective, molecular epidemiology study of EGFR mutations in Asian patients with advanced non-small-cell lung cancer of adenocarcinoma histology (PIONEER). J Thorac Oncol 9: 154-162, 2014.

38. Riely GJ, Pao W, Pham D, et al: Clinical course of patients with non-small cell lung cancer and epidermal growth factor receptor exon 19 and exon 21 mutations treated with gefitinib or erlotinib. Clin Cancer Res 12: 839-844, 2006.

39. Sun JM, Won YW, Kim ST, et al: The different efficacy of gefitinib or erlotinib according to epidermal growth factor receptor exon 19 and exon 21 mutations in Korean non-small cell lung cancer patients. J Cancer Res Clin Oncol 137: 687-694. 2011.

40. Tam IY, Chung LP, Suen WS, et al: Distinct epidermal growth factor receptor and KRAS mutation patterns in non-small cell lung cancer patients with different tobacco exposure and clinicopathologic features. Clin Cancer Res 12: 1647-1653, 2006.

41. Marks JL, Broderick S, Zhou Q, et al: Prognostic and therapeutic implications of EGFR and KRAS mutations in resected lung adenocarcinoma. J Thorac Oncol 3: 111-116, 2008

42. Yano M, Sasaki H, Kobayashi Y, et al: Epidermal growth factor receptor gene mutation and computed tomographic findings in peripheral pulmonary adenocarcinoma. J Thorac Oncol 1: 413-416, 2006

43. Yoshida Y, Kokubu A, Suzuki K, et al: Molecular markers and changes of computed tomography appearance in lung adenocarcinoma with ground-glass opacity. Jpn J Clin Oncol 37: 907-912, 2007.

44. Chung JH, Choe G, Jheon S, et al: Epidermal growth factor receptor mutation and pathologic-radiologic correlation between multiple lung nodules with ground-glass opacity differentiates multicentric origin from intrapulmonary spread. J Thorac Oncol 4: 1490-1495, 2009 
45. Glynn C, Zakowski MF and Ginsberg MS: Are there imaging characteristics associated with epidermal growth factor receptor and KRAS mutations in patients with adenocarcinoma of the lung with bronchioloalveolar features? J Thorac Oncol 5: 344-348, 2010.

46. Park EA, Lee HJ, Kim YT, et al: EGFR gene copy number in adenocarcinoma of the lung by FISH analysis: investigation of significantly related factors on CT, FDG-PET, and histopathology. Lung Cancer 64: 179-186, 2009.

47. Lee Y, Lee HJ, Kim YT, et al: Imaging characteristics of stage I non-small cell lung cancer on CT and FDG-PET: relationship with epidermal growth factor receptor protein expression status and survival. Korean J Radiol 14: 375-383, 2013.
48. Hsu KH, Chen KC, Yang TY, et al: Epidermal growth factor receptor mutation status in stage I lung adenocarcinoma with different image patterns. J Thorac Oncol 6: 1066-1072, 2011.

49. Aoki T, Hanamiya M, Uramoto H, Hisaoka M, Yamashita Y and Korogi Y: Adenocarcinomas with predominant ground-glass opacity: correlation of morphology and molecular biomarkers. Radiology 264: 590-596, 2012.

50. Riely GJ, Politi KA, Miller VA and Pao W: Update on epidermal growth factor receptor mutations in non-small cell lung cancer. Clin Cancer Res 12: 7232-7241, 2006. 